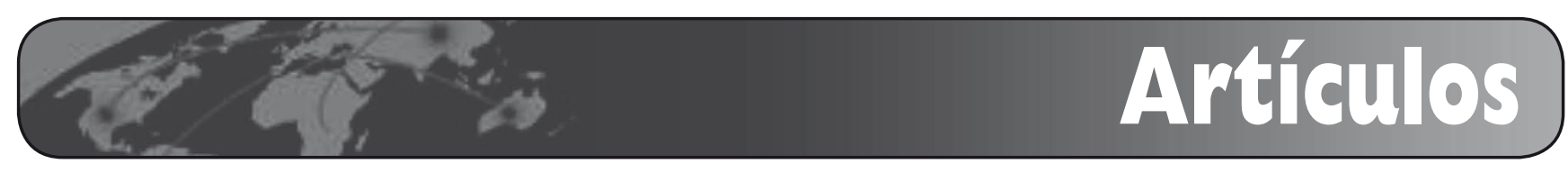

\title{
Web móvil y bibliotecas
}

\author{
Por Natalia Arroyo-Vázquez
}

\begin{abstract}
Resumen: El acceso a la Web desde dispositivos móviles (teléfonos móviles, pdas, smartphones, ultraportátiles, portátiles de bajo coste, etc.) presenta ventajas como la movilidad y la consulta de información y servicios desde cualquier punto con una conexión a internet, pero también plantea nuevas cuestiones relacionadas con la accesibilidad de los contenidos, basadas fundamentalmente en las peculiaridades físicas de esos dispositivos y en sus conexiones (pantallas pequeñas, teclados diferentes a los de los ordenadores convencionales, limitaciones de memoria y formatos soportados, o conexiones más lentas), pero también en el contexto de movilidad en que se producen, lo que genera unas necesidades diferentes. En el presente artículo se aporta una visión general sobre la

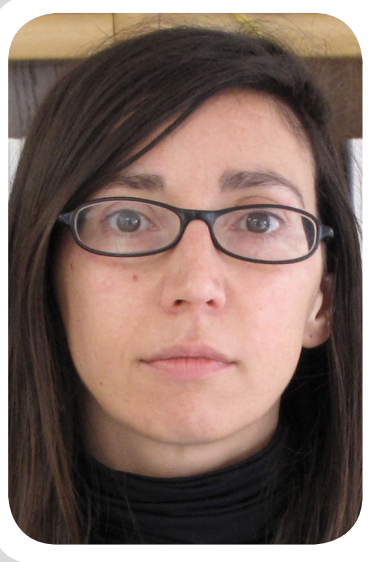
Natalia Arroyo-Vázquez es licenciada en documentación por la Universidad de Salamanca, grado de Salamanca y diploma de estudios avanzados. Desde hace cuatro años trabaja como documentalista en el Departamento de Análisis y Estudios de la Fundación Germán Sánchez Ruipérez. Compagina su trabajo habitual con la docencia sobre temas relacionados con la aplicación de los servicios de la Web social en las bibliotecas, tema sobre el que ha publicado varios artículos en publicaciones especializadas así como comunicaciones a jornadas y congresos.
\end{abstract}

Web móvil, cómo afecta a las bibliotecas y cómo pueden actuar éstas en consecuencia.

Palabras clave: Web, Web móvil, Accesibilidad, Teléfonos móviles, Dispositivos móviles, Bibliotecas.

\section{Title: Libraries and the mobile web}

Abstract: Mobility and access to Web contents and services from any place having an internet connection are both advantages of using mobile devices like mobile phones, pdas, smartphones, ultra mobile PCs or ultra low cost PCs. However, new questions also arise, mainly related to content accessibility: their characteristics, connections and the way people in motion connect to the internet generate different requirements. In this paper a general landscape of the mobile web is drawn from a librarian's perspective.

Keywords: Web, Mobile web, Accessibility, Mobile phones, Mobile devices, Handheld, Libraries.

Arroyo-Vázquez, Natalia. "Web móvil y bibliotecas”. El profesional de la información, 2009, marzo-abril, v. 18, n. 2 , pp. 129-136.

DOI: 10.3145/epi.2009.mar.02

\section{La Web móvil}

El avance de las telecomunicaciones, capaces de permitir las conexiones inalámbricas y las consultas a internet desde el teléfono móvil, y la aparición en el mercado de una gran variedad de dispositivos cada vez más fáciles y cómodos de usar, en los que la conectividad es un extra más, llevan a acuñar la expresión Web móvil para referirse al acceso a la Web desde aparatos cuya principal cualidad es la movilidad. Se trata de la misma Web que consultamos desde nuestros ordenadores de sobremesa o portátiles, el único elemento diferenciador es el equipo empleado (Kroski, 2008). Algunos autores prefieren definir la Web móvil no desde el punto de vista del equipo, sino del contexto de movilidad en clara referencia al usuario (Ballard, 2007).
El número de personas que accede mediante móviles es aún reducido: en España y en el año 2007 sólo un 20\% de los internautas utilizó esta forma de acceso -un 10\% desde una pda, el $12 \%$ desde una videoconsola, y casi un $2 \%$ desde otros equipos (Navegantes en la red, 2008)Frente a ellos, un $92 \%$ lo hace desde un ordenador de sobremesa y el $60 \%$ desde un ordenador portátil. Sin embargo la mayor parte de los expertos coincide en que ésta será la principal forma de acceso en los próximos diez años (Quitney; Rainie, 2008), como ya sucede en Japón. Por el momento se están dando varios factores que pueden propiciar el cumplimiento de esa predicción: la penetración de la telefonía móvil en España es muy alta, de un $111,9 \%$, y el $20 \%$ de los usuarios ya tiene una teléfono 3G (tecnología comparable a la banda ancha), uno de los porcentajes más altos de Europa (eEspaña, 2008). 
Pero no todos los dispositivos afectados son teléfonos ni todas las conexiones se realizan mediante las redes de telefonía: la aparición en 2008 de los primeros ordenadores portátiles de bajo coste (ultra low cost $P C$ o $u l c p c$ ), inspirados en el proyecto One Laptop per Child, está suponiendo un éxito, ya que vienen a cubrir las necesidades de un sector de la población con una alta movilidad y de quienes buscan una solución barata. Por otra parte, se está desarrollando una amplia y variada gama de gadgets que incluyen la conectividad como una prestación complementaria, como es el caso de videoconsolas, iPods y libros electrónicos.

Parecen ser los usuarios frecuentes de internet, que en España son un 49,2\% en $2008^{2}$, quienes están abanderando este cambio (eEspaña, 2008): nos acostumbramos a disponer de información y de una serie de servicios que nos facilitan algunas tareas de la vida cotidiana, o que simplemente nos divierten, y queremos tenerlos allí donde nos encontremos.

\section{"El término Web móvil hace referencia al acceso a la Web desde aparatos cuya principal cualidad es la movilidad"}

Uno de los elementos que están contribuyendo a la difusión de la Web móvil son las redes sociales ( $L a$ sociedad..., 2008), que desde hace un tiempo brindan la posibilidad de estar conectados con otras personas y compartir recursos con ellas de forma instantánea: desde nuestro teléfono móvil, por ejemplo, podemos publicar un texto en nuestro blog, subir a Flickr o YouTube las fotos o vídeos que acabamos de tomar con su cámara, o comunicarnos mediante Twitter, Facebook o Tuenti con nuestros contactos, y todo ello mediante interfaces creadas específicamente para ello ${ }^{3}$.

Se habla también de una Web móvil 2.0 (Jaokar; Fish, 2006), que es la materialización de uno de los principios de la Web social descritos por O'Reilly (2005): el software (incluida cualquier aplicación web) no se limita a un solo dispositivo. Así, la Web móvil 2.0 no es más que la Web 2.0 en un contexto de movilidad, lo que conlleva el uso de aparatos móviles no sólo para buscar información, sino también para interactuar con otras personas en las redes sociales o agregar contenidos en sitios para compartir información. En palabras de Jaokar y Fish (2006), la Web móvil 2.0 es una extensión de la Web que se centra en el usuario como creador y consumidor de contenidos "en el punto de inspiración"; el dispositivo móvil es el medio para alcanzar la inteligencia colectiva.

\footnotetext{
Web móvil $=$ contenidos $(1.0)+$ contacto $(2.0)$
}

Ni que decir tiene que la Web móvil afecta a todos los sectores con contenidos web y a quienes suministran los servicios y, por consiguiente, también a las bibliotecas. En los próximos apartados trataremos de abordar de forma general y desde el punto de vista del creador de contenidos los diferentes elementos a tener en cuenta en la Web móvil y cómo pueden actuar en consecuencia las bibliotecas.

\section{"La Web móvil cambia los hábitos y la forma de acceso a la información"}

\section{Los dispositivos móviles}

En la Web móvil intervienen varios agentes, desde las redes de telecomunicaciones o los proveedores del servicio (operadores de telefonía móvil) hasta los equipos empleados para la visualización de los contenidos. Estos últimos merecen una atención especial para quienes están interesados en la producción de contenidos, puesto que de sus posibilidades depende la experiencia que el usuario final pueda obtener.

El $W 3 C$ define el término dispositivo móvil como un "aparato portátil, con el que se puede acceder a la Web y diseñado para ser usado en movimiento" (Nkeze; Pearce; Womer, 2007). Este concepto abarca por lo tanto una amplia variedad de terminales con cualidades muy diferentes, lo que complica el proceso de adaptación de contenidos, como se verá más adelante. Se trata además de un mercado cambiante donde cada año surgen novedades y mejoras tecnológicas.

Si bien los móviles aportan movilidad en la consulta de la Web para sus propietarios, además de una mayor conectividad y acceso a la información, también presentan varias limitaciones derivadas de las redes de comunicaciones, como la dependencia de una conexión telefónica o de una red wifi abierta, o marcadas por los propios dispositivos y el software, como son las restricciones en la visualización de los contenidos y la interacción. Veamos a continuación algunas de ellas (Arroyo, 2008), puesto que deberán ser tenidas en cuenta a la hora de elaborar contenidos.

- Pantallas pequeñas, entre las 2 pulgadas de un teléfono móvil y las 10 de un ordenador ultraportátil.

- Teclados de reducidas dimensiones y no qwerty, como los de los teléfonos móviles, o accionados por el tacto, punteros/lápices o botones de posición.

- Ausencia de ratón y/o puntero, por lo que deben emplearse las teclas de posición para la navegación. 
- Reducida capacidad de memoria, lo que coarta el uso de cookies y caché.

- Algunos equipos no soportan JavaScript o Flash, o ciertos formatos de vídeo.

- La carga de páginas más pesadas se puede ver entorpecida por conexiones lentas.

- Algunos contratos de telefonía establecen un pago por cantidad de datos descargados, por lo que las grandes descargas encarecerán el servicio.

En el mercado encontramos una gran variedad de teléfonos móviles con conexiones de datos cada vez más rápidas y que oscilan entre los $14,4 \mathrm{~Kb}$ por segundo de la tecnología GSM y los 7,2 Mbps en bajada de la tecnología Hsupa (High-speed uplink packet access). En este gran grupo se incluyen las pda (personal digital assistant) -pequeños ordenadores que funcionan a modo de agendas electrónicas-, y los smartphones o teléfonos inteligentes -que son móviles con sistema operativo incorporado, lo que les habilita para ejecutar programas ofimáticos-. Sus pantallas son algo mayores que las de los teléfonos móviles (en torno a unas 3 pulgadas) y pueden incorporar un pequeño teclado alfanumérico similar al de un ordenador. Algunos incorporan también wifi y sus propiedades varían enormemente de unos fabricantes a otros, al igual que los sistemas operativos que emplean. Además de las Blackberry, Motorola $Q$ y otros, el terminal más popular actualmente es el iPhone de Apple, que ha puesto de moda las pantallas táctiles y las aplicaciones.

\section{"Los expertos coinciden en que la principal forma de acceso a internet en los próximos diez años será móvil"}

Otro tipo de dispositivos móviles son las videoconsolas portátiles, como la PSP (PlayStation Portable) de Sony, una de las más populares en nuestro país, con una pantalla panorámica a color de 4,3 pulgadas. Se maneja con ambas manos, pues tiene botones a derecha e izquierda que sirven para accionar un teclado virtual y activar el navegador ante la ausencia de ratón. La conexión a internet es wifi.

El lanzamiento del lector-e Kindle de Amazon ha abierto las posibilidades de mercado para un nuevo tipo de dispositivo, el libro electrónico, cuya finalidad última es la lectura de textos. Aunque son varios los que disponen de conexión vía wifi, como el iRex Iliad de Philips, FLEPia de Fujitsu, el Kindle de Amazon o Readius (Monteoliva; Pérez-Ortiz; Repiso, 2008), su única finalidad suele ser la descarga de libros, la consulta de determinadas páginas o la actualización de noticias mediante RSS, y no la navegación, aunque no cabe descartar que ésta sea una de las opciones que se hagan realidad en el futuro, en la línea marcada por el pionero Readius, el teléfono lector de documentos desplegable.

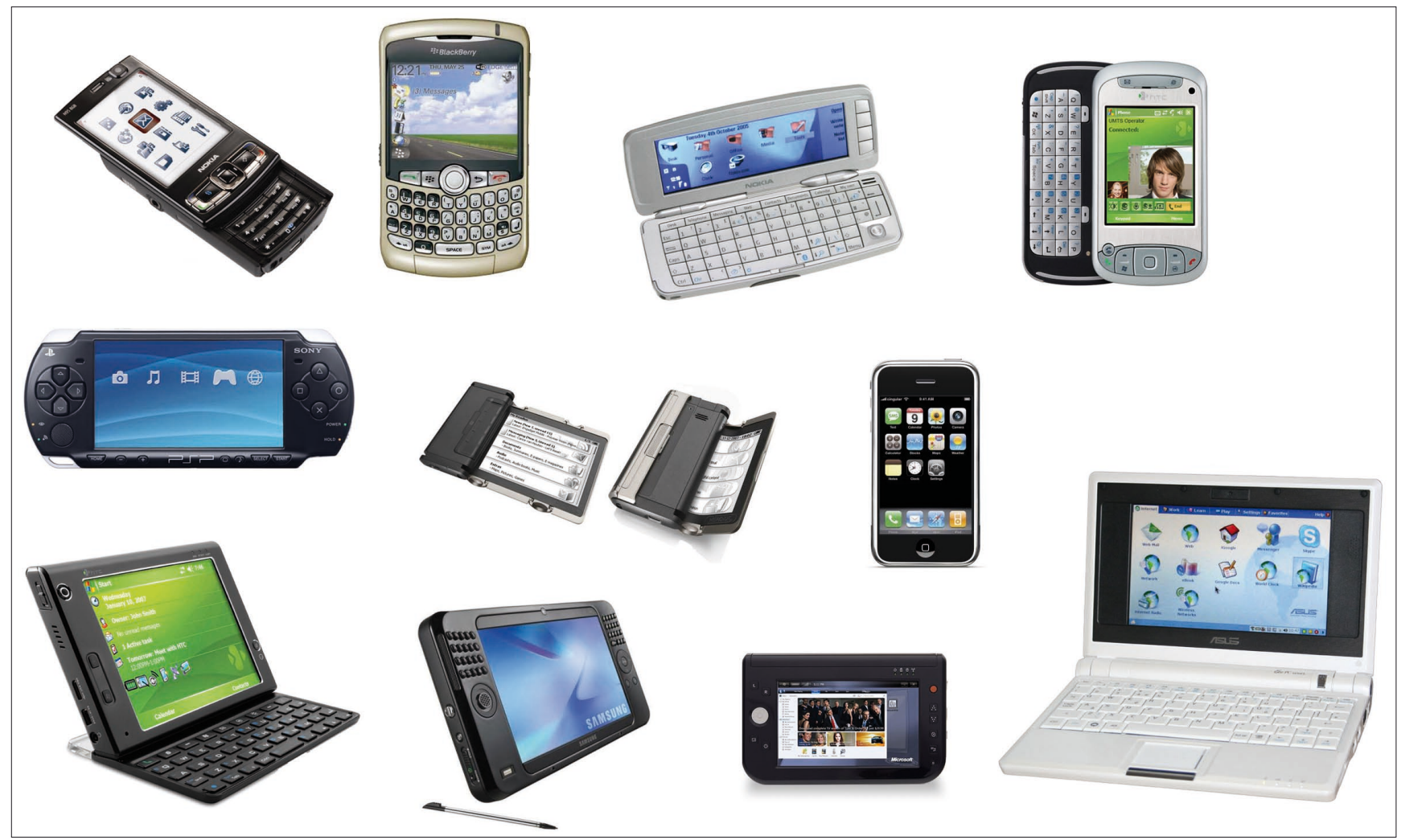

Diferentes tipos de dispositivos móviles 
Hay una gran variedad de pequeños ordenadores con múltiples denominaciones y diferentes propiedades, pensados para cubrir necesidades diversas. Ultramobile PC (umpc) es una denominación que se usa popularmente para designar a una gama de ordenadores de pequeñas dimensiones (netbooks, notebooks...) . $^{4}$ Sus capacidades se acercan cada vez más a las de un ordenador portátil, alcanzan 1GB de RAM, discos duros de 80GB, y algunos incluyen el mismo software que los ordenadores portátiles y de sobremesa.

\section{"Los equipos móviles presentan limitaciones debidas a su formato, a las redes de comunicaciones y al software"}

El precio de los umpc hace que no se hayan popularizado de la misma manera que los ultra low cost $P C$ (ulcpc), ordenadores portátiles de pequeñas dimensiones y bajo coste (alrededor de $350 €$ ). Están diseñados para conectarse a internet y realizar tareas de ofimática, entretenimiento, etc., y sus atributos son algo inferiores a los de un ordenador portátil, aunque están evolucionando. El precursor, con gran éxito, ha sido el Asus $E e e P C$, al que luego han seguido otros fabricantes.

Estos pequeños ordenadores tienen menos limitaciones que el resto de los aparatos descritos: sus prestaciones y software suelen ser muy similares a los de cualquier ordenador portátil, aunque sus dimensiones son mucho menores (hasta 10 pulgadas de pantalla), con lo es posible visualizar sitios web sin demasiadas dificultades, aunque con algunas diferencias que no lo hacen óptimo para navegar durante largos espacios de tiempo. Es por ello que cuando se piensa en adaptar contenidos para móviles no suelen ser tenidos en cuenta.

\section{Adaptar sitios web para móviles}

Aunque cualquier web puede ser visualizada desde cualquier dispositivo móvil con navegador, si queremos mejorar la experiencia de navegación del usuario puede que sea precisa una adaptación de los contenidos. El World Wide Web Consortium (W3C) la define como "un proceso de selección, generación o modificación que produce contenidos en respuesta a la petición de una URI en un contexto de presentación determinado. Este contexto incluye las capacidades del dispositivo, así como un conjunto de atributos que caracterizan a las capacidades del mecanismo de acceso y las preferencias del usuario" (Nkeze; Pearce; Womer, 2008).

El proceso de adaptación plantea algunas cuestiones previas. La principal reside en decidir si modificar las páginas de mi sitio web para su consulta desde

\section{"Algunas bibliotecas han creado ya interfaces de su catálogo pensando en su consulta desde móviles"}

dispositivos móviles y de qué manera hacerlo (Moll, 2008). Para ello podemos tener en cuenta quiénes son nuestros usuarios, si acceden a la web mediante este tipo de equipos y si les gustaría o no que la biblioteca ofreciera servicios optimizados para ellos. Sin embargo podemos barajar la posibilidad de que a otras personas que no son usuarios nuestros también les pueda interesar la información que podamos suministrarles, de manera que podríamos adelantarnos a sus necesidades.

La primera solución ante la disyuntiva de si adaptar o no nuestro sitio web sería no hacer nada, ante la consideración de que quienes acceden a nuestros contenidos emplean ordenadores de sobremesa o portátiles, siendo aún mínimas las consultas desde terminales dotados de movilidad. En todo caso, quienes consultan la web desde los mismos pueden arreglárselas de diferentes maneras: los navegadores tienen opciones para visualizar la información, como el zoom o el scrolling. Además, hay soluciones externas que nuestros usuarios pueden adoptar, como el empleo de servicios web gratuitos que realizan este trabajo automáticamente, como Skweezer, Mowser o el servicio de Google.

\section{http://www.skweezer.com/ \\ http://mowser.com/ \\ http://www.google.com/gwt/n}

Una solución intermedia pasa por enlazar desde

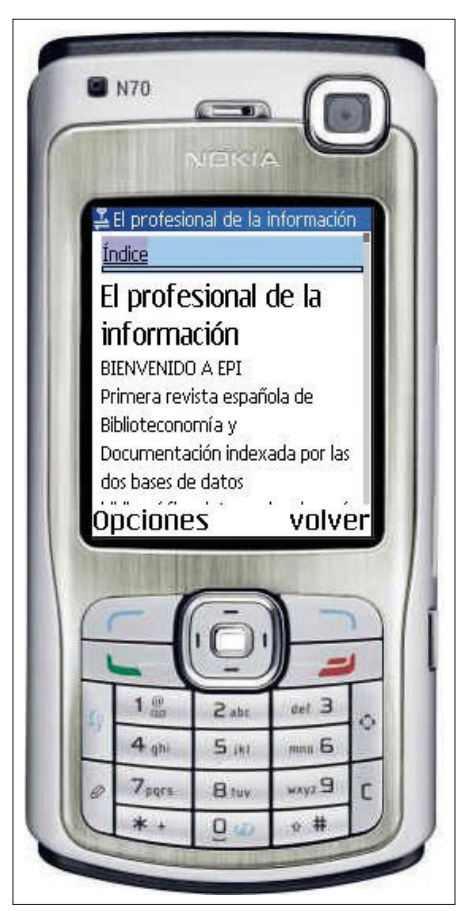

El profesional de la información nuestro sitio web la URL resultante de la conversión de nuestra web mediante alguno de esos servicios anteriormente citados ${ }^{5}$. También se pueden crear diferentes hojas de estilo que cambien la visualización de los contenidos de nuestro sitio, manteniendo intacta toda la información.

La existencia de una gran variedad de aparatos en el mercado puede llevarnos a cuestionarnos para qué tipo optimizar nuestro sitio web. Sin duda al- 
guna lo mejor sería que pudiera ser visto sin problema alguno desde cualquiera de ellos, aunque esto puede entrañar una mayor complicación. Algunas entidades han optado incluso por crear diferentes hojas de estilo que se activan al ser conectadas por un determinado terminal.

Y, finalmente, en el caso de que decidamos crear un nuevo sitio web para dispositivos móviles, ¿cómo hacerlo?, ¿qué contenidos y/o servicios facilitar?, ¿los mismos o diferentes? El W3C se muestra a favor del principio "One web" (Rabin; McCathieNevile, 2008), lo que significa poner a disposición de los usuarios de terminales móviles la misma información y servicios que los ordenadores de escritorio, en la medida de lo posible. Esto no significa que la información y su representación sean exactamente las mismas para todos los dispositivos, ya que las cualidades de éstos y el contexto en que son empleados son diferentes, y por lo tanto habrá servicios e información más apropiados para unos contextos que para otros. Se trata de preguntarse por qué a alguien le interesaría consultar nuestro sitio web en una situación de movilidad y cuál es el tipo de información o servicios que le pueden interesar: lo más lógico es que trate de obtener datos puntuales que le puedan ser de utilidad práctica y no navegar por un sitio web en busca de una gran cantidad de datos.

A la hora de generar una web para dispositivos móviles podemos emplear html, pero hay lenguajes estándar para tal fin como $x$ tml mobile profile (xhtm-mp) y css mobile profile 2.0 para hojas de estilo.

El Best practices working group (BPWG) del W3C, en el marco de la Mobile web initiative, ha elaborado una lista de buenas prácticas para la publicación de contenidos web para móviles, las Mobile web best practices (Rabin; McCathieNevile, 2008). En este sentido, el $W 3 C$ ha redactado también una serie de principios para garantizar la independencia de los aparatos, de contenidos y aplicaciones web, los Device independence principles (Gimson, 2003), que afectan también a este ámbito.

http://www.w3.org/Mobile/

A la hora de diseñar un sitio web para terminales móviles se recomienda de forma general ${ }^{6}$ tener en cuenta sus limitaciones, vistas en el capítulo anterior. Así, es preciso crear sitios web de tamaño reducido, pensados para ser vistos en pequeñas pantallas, y aligerar la información al máximo haciéndola lo más concisa posible y esencialmente textual, evitando imágenes pesadas y objetos multimedia, así como formatos como JavaScript o Flash. Como ayuda puede servirnos el test del $W 3 C$ basado en las Mobile web best practices, el mobileOK checker o emuladores como el de dot.mobi.

http://validator.w3.org/mobile/ http://mtld.mobi/emulator.php

\section{La Web móvil y las bibliotecas}

Las bibliotecas, como proveedoras de contenidos y servicios web, pueden plantearse la optimización para dispositivos móviles. Ante esta propuesta surgen varias cuestiones, que ya han sido abordadas en el apartado anterior, y distintas posibilidades que veremos a continuación, para trasladar los servicios ya existentes y brindar otros nuevos, todo ello a la luz de algunos ejemplos. Megan Fox en su web y en sus presentaciones para varias conferencias (Fox, 2007) nos ofrece muchos otros casos de bibliotecas en Norteamérica que nos pueden servir como modelos además de proporcionarnos una gran cantidad de recursos.

\section{Sedes web}

Algunas bibliotecas han decidido adaptar los contenidos de sus webs para una mejor accesibilidad desde dispositivos móviles, bien mediante servicios gratuitos con la ventaja de no necesitar conocimientos avanzados -como es el caso de la Freemont Public Library, Mundelein, Illinois (EUA), que ha usado mobiSiteGalore-, o bien elaborando páginas específicas. Es el caso de la Harris County Public Library, Houston, Texas (EUA), que ha creado un sencillo sitio en un html muy básico, o de la Regina Public Library, Regina, Saskatchewan (Canadá), cuya homepage reenvía directamente a sus sitios web para móviles, o de la Ball State University Library, Muncie, Indiana (EUA) (West, 2007).

\section{http://fpld.websiteforever.mobi/ \\ http://websiteforever.mobi/ \\ http://pda.hcpl.net/ \\ http://www.reginalibrary.ca/m/ \\ http://www.bsu.edu/libraries/mobile/}

Todos ellos han optado fundamentalmente por mostrar información básica que a alguien en movilidad le resultaría útil conocer de una forma rápida y sencilla, como pueden ser los datos de contacto y horario de la biblioteca, servicios que ofertan y los próximos eventos, todos ellos expresados de la forma más breve y concisa posible. La Harris County Public Library proporciona además listados de novedades y de los libros más prestados, FAQs y acceso al catálogo y gestiones personales como renovaciones. Sus contenidos son algo más extensos. El diseño es limpio y muy sencillo: fondo blanco, sin imágenes, información organizada en listados y poca en cada página.

También hay contenidos que no necesitan ser adaptados, como los distribuidos mediante servicios de la Web 2.0 como Twitter, Facebook o YouTube, por citar sólo algunos, ya que todos ellos disponen de interfaces específicas, como se señaló en el primer apartado de este texto. Los sitios web con contenidos sindicados, como los blogs, pueden ser vistos con agregadores especiales para iPhone, pdas o teléfonos móviles, como 


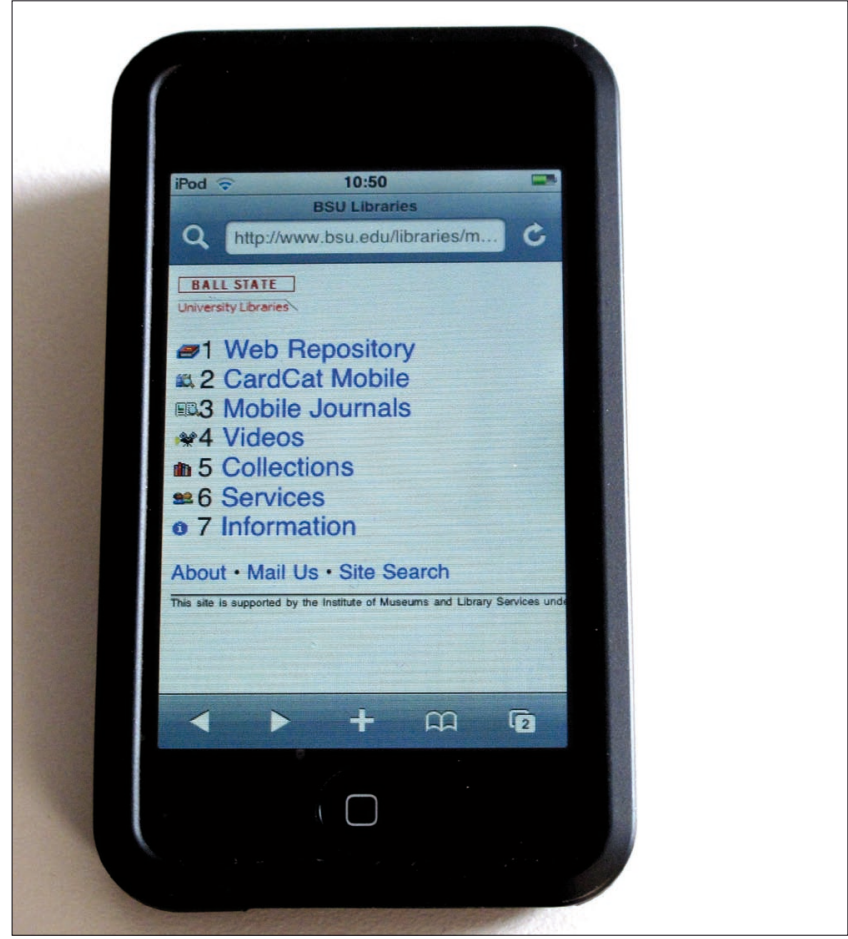

Ball State University Libraries

Feedalot (Farkas, 2007). Es lo que ha hecho la Biblioteca Municipal de Muskiz adecuando los contenidos del boletín Bateginik, todos ellos en forma de blog, para ser leídos desde PSP con el servicio gratuito $x$ Fruits.

http://xfruits.com/ferjur/?id=13423

\section{Catálogos móviles (mopacs)}

La consulta de bases de datos en línea puede complicarse desde un dispositivo móvil de pequeña pantalla: la ausencia de ratón dificulta la selección de enlaces o de opciones en los menús y la escritura puede hacerse engorrosa desde pequeños teclados no qwerty. Los resultados suelen presentarse en largos listados y de cada registro se obtiene gran cantidad de información, lo que obliga a desplazarse por la página en una pantalla pequeña.

Para solucionar estos inconvenientes algunas bibliotecas han creado interfaces de su catálogo pensando en su consulta desde móviles. A estos catálogos se les ha llamado en la literatura anglosajona mopacs, por mobile opacs (Kroski, 2008). El resultado son catálogos con información concisa e interfaces de búsqueda sencillas que evitan las tablas y el diseño gráfico, y que responden simplemente a las necesidades que un usuario pueda tener en movimiento: consultar la existencia de un documento en la biblioteca, su ubicación y disponibilidad. No se trata de crear nuevas bases de datos, sino de confeccionar nuevas interfaces que muestren los contenidos de las ya existentes de manera apropiada.

En este sentido tenemos soluciones de empresas como AirPAC, de Innovative Interfaces, que forma par- te del sistema integrado Millenium (Eíto, 2008), adoptado también por algunas bibliotecas universitarias españolas. Mediante AirPAC un usuario puede tener acceso cómodamente al catálogo desde una pequeña pantalla y ver la información de su carnet de la biblioteca, gestionar reservas o renovar préstamos. Algunas de las bibliotecas que emplean este software son la Hennepin County Library, Minneapolis, Minnesota (EUA) y la biblioteca de la Universidad de Cádiz, España.

http://mplwebcat.mplib.org/airpac/ http://biblioteca.uca.es/diana/Airpac.asp

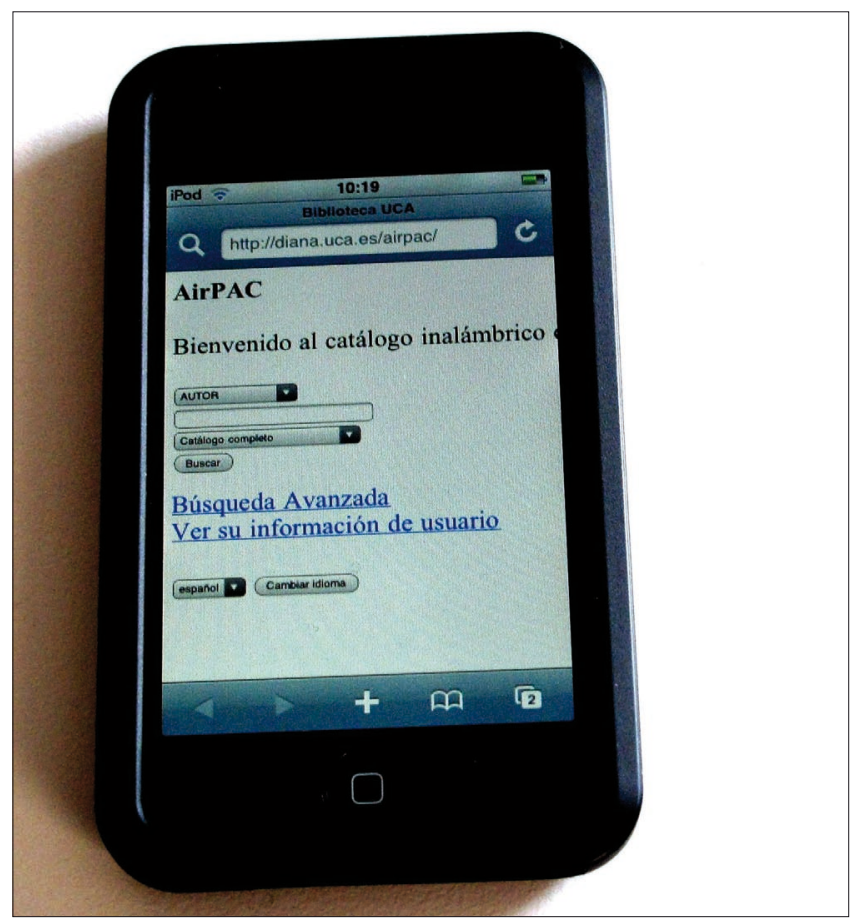

Catálogo AirPAC de la Universidad de Cádiz

MobiLIB es una interfaz confeccionada para la visualización del catálogo de las bibliotecas de la North Carolina State University, Raleigh, North Carolina (EUA), en dispositivos móviles (Sierra; Ryan; Wust, 2008). Opera sobre la plataforma CatalogWS y surge con la filosofía de no limitar la consulta del catálogo a una sola aplicación y superando el reto de ser apto para varios tipos de equipos móviles.

\section{http://www.lib.ncsu.edu/m/catalog/}

El servicio de la web social LibraryThing, que facilita la creación de un catálogo personal en línea y que se ha convertido en uno de los ejemplos a imitar por los llamados opac 2.0, posibilita también la consulta a nuestro catálogo desde un dispositivo móvil y ver la información básica de los registros que hemos guardado: título, datos bibliográficos, etiquetas, posibles vendedores y una pequeña imagen de la portada.

http://www.librarything.com/m/ 


\section{Aplicaciones específicas para móviles}

Además de acceso a la web, los móviles brindan nuevas posibilidades para las bibliotecas, entre las que se encuentran aplicaciones de geoposicionamiento (Tosete, 2007) y la lectura de libros electrónicos, además de ser capaces de reproducir música y vídeos. Las conexiones, cada vez más potentes, posibilitan descargas de grandes documentos.

Hay programas lectores de libros electrónicos, como MobiPocket, que podemos descargar de forma gratuita e instalar en nuestra pda. Está disponible para varios sistemas operativos (Blackberry, Symbian, Windows Mobile, Palm), incluso para PC y lectores de libros electrónicos (viene preinstalado en Cybook e iLiad). El sistema de descargas es idéntico al de $i T u$ nes, incluso su programa lo recuerda visualmente: se pueden descargar los libros de su catálogo mediante el propio programa y se paga por cada documento descargado. El coste de los libros es mucho menor que el de un libro impreso, algunos pueden costar lo mismo que una politono o música de móvil, otros son incluso gratuitos.

\section{http://www.mobipocket.com/}

Una de las novedades tecnológicas del pasado año fueron las aplicaciones para iPhone e iPod Touch, entre las cuales hay dos lectores de libros electrónicos: Stan$z a$ y e-Reader, este último también disponible para muchos otros dispositivos, y ya se anuncian dos nuevas: Shortcovers e Iceberg (Mossberg, 2009).

\section{http://www.lexcycle.com/}

http://www.ereader.com/

Algunas bibliotecas públicas facilitan servicios de descargas de libros electrónicos a sus usuarios con intermediarios comerciales. Es el caso de la Biblioteca Pública de Nueva York, que mediante la empresa especializada OverDrive ofrece a sus usuarios préstamos de libros electrónicos y audiolibros, música y vídeos descargables.

\section{http://ebooks.nypl.org/}

Sin necesidad de contratar intermediarios las bibliotecas pueden facilitar el acceso a libros electrónicos gratuitos mediante enlaces a bibliotecas virtuales como el Proyecto Gutenberg o Google Books, o como la Mansfield/Richland County Library, Ohio (EUA), que muestra este tipo de documentos, o incluso hacer sus propios libros-e accesibles desde estos aparatos.

\section{http://www.gutenberg.org/} http://books.google.com/m http://www.mrcpl.lib.oh.us/PDA_e_books.htm

Volviendo una vez más al mundo de las aplicaciones y a modo de curiosidad, podemos encontrar una aplicación para el iPhone de la District of Columbia Public Library, Washington, DC. Desde ella es posible consultar los horarios de cada una de sus sedes, buscar en el catálogo y hacer reservas en línea.

http://www.walkingpaper.org/1100

\section{Conclusiones y discusión}

El debate sobre la prestación de servicios bibliotecarios mediante dispositivos móviles y la adaptación de contenidos en ese mismo entorno está servido. Todo lo dicho en los anteriores apartados lleva a plantearse una serie de preguntas: ¿es oportuno adaptar los contenidos ofrecidos por las bibliotecas vía web para ser leídos desde estos aparatos? ¿Cuándo hacerlo? ¿Por qué y con qué propósito? ¿Para quién? ¿Cómo abordar el problema?

El acceso a la Web desde equipos móviles no está aún consolidado, sino que se encuentra en un estadio incipiente. Las aplicaciones de estos aparatos están en plena elaboración: el número de usuarios es aún reducido y el mercado tecnológico está en ebullición, con constantes lanzamientos de nuevos y mejores terminales con más prestaciones. Este escenario inicial, aunque con bastantes expectativas de futuro, es propicio para la iniciación y la realización de pruebas.

No hay que olvidar la realidad de cada biblioteca, sus entornos interno y externo y el tipo de usuarios a los que sirve. Las bibliotecas universitarias (también las especializadas) pueden encontrar más posibilidades en un primer momento, dado que tanto alumnos como docentes pueden acercarse más al perfil de usuarios de la Web móvil: jóvenes de nivel económico medioalto y muy familiarizados con las tecnologías móviles y las redes sociales, así como docentes de alto nivel económico, con más posibilidades de conocer las tecnologías, con una alta movilidad y la necesidad de estar conectados. Las bibliotecas públicas y escolares también tienen ante sí la oportunidad de ofrecer contenidos de interés práctico o servicios adicionales, algo que, si se cumplen las expectativas de los expertos, probablemente se difunda ampliamente en un futuro.

A modo de resumen, se muestran a continuación algunas pautas a tener en cuenta a la hora de abordar un proceso de adaptación. En primer lugar y a la hora de decidir si adaptar nuestros contenidos y servicios o no, puede ser de ayuda conocer el número de usuarios de la biblioteca que emplean dispositivos móviles para acceder a internet, a quiénes les interesaría que la biblioteca ofreciera servicios móviles y qué dispositivos emplean para ello: recordemos que la experiencia de navegación varía enormemente al usar un móvil normal o una pda, por citar sólo un par de ejemplos. Otra posibilidad es adelantarse a los acontecimientos para 
conocer cuál sería la respuesta de nuestros usuarios o de otros que no lo sean. En este caso es interesante la siguiente recomendación:

Para comenzar pueden adoptarse soluciones sencillas, como emplear servicios web gratuitos, con dos finalidades: familiarizarnos con el entorno móvil y probar la aceptación del servicio. Esto no es un esfuerzo demasiado grande y puede permitir la posterior implementación de otros servicios más avanzados y costosos.

Ponerse en el lugar de quien necesita esa información es sin duda el primer ejercicio a realizar antes de decidir qué servicios ofrecer. Mostrar toda la información que tenemos en nuestra web no tiene demasiado sentido y puede dificultar la navegación y la localización de los contenidos esenciales.

La Web móvil ofrece la posibilidad de romper las barreras físicas de la biblioteca, como ya ha demostrado internet, pero además promete cambiar los hábitos y la forma de llegar a la información, cada vez más accesible desde cualquier lugar, y por lo tanto también la forma de dispensar los servicios.

\section{Notas}

1. Según el último informe mensual publicado por la Comisión del Mercado de las Telecomunicaciones (CMT) correspondiente a octubre de 2008.

http://www.cmt.es/es/publicaciones/anexos/081205_NM_octubre08.pdf

2. Instituto Nacional de Estadística (INE).

http://www.ine.es/

3. Las interfaces específicas para móviles de Flickr, YouTube, Twitter, Facebook y Tuenti están disponibles en los siguientes URLs:

http://m.flickr.com/

http://m.youtube.com/

http://m.twitter.com/

http://m.facebook.com/

http://m.tuenti.com/

4. Este término ha sido empleado por Microsoft en el marco de su Proyecto Origami para designar a los ordenadores de mano, de pequeñas dimensiones, con pantallas entre 4 y 7 pulgadas y un peso inferior a 1 kilo, pantalla táctil y algunos con sistemas de escritura con teclado y/o punteros.

5. En el caso de la revista El profesional de la información y empleando el servicio de Google sería

http://www.google.com/gwt/n? $u=h t t p \% 3 A \% 2 F \% 2 F w w w . e l p r o f e s i o n a l d e$ lainformacion.com $\% 2 F \& \_g w t$ noimg $=1$

6. Para un mayor nivel de detalle consultar la documentación citada en el párrafo anterior.

\section{Bibliografía}

Arroyo-Vázquez, Natalia. "Accesibilidad de los contenidos en internet de las bibliotecas públicas desde dispositivos móviles". Anuario ThinkEPI 2009. Barcelona: EPI SCP, 2009.

http://www.thinkepi.net/accesibilidad-de-los-contenidos-en-internet-delas-bibliotecas-publicas-desde-dispositivos-moviles

Ballard, Barbara. Designing the mobile user experience. Wiley, 2007. ISBN: 978-0-470-03361-6.

eEspaña 2008: informe anual sobre el desarrollo de la sociedad de la información en España. Madrid: Fundación Orange, 2008.

http://www.fundacionorange.es/areas/25_publicaciones/e2007.pdf
Eíto-Brun, Ricardo. "Sistemas integrados para bibliotecas públicas. Las propuestas de Innovative". El profesional de la información, 2008, marzoabril, v. 17, n. 2, pp. 225-230.

Farkas, Meredith. "The mobile revolution". En: Farkas, M. Social soft ware in libraries: building collaboration, communication and community online. Medford: Information Today Inc, 2007, pp. 167-180. ISBN 978-157387-275-1.

Fox, Megan K. Pdas, handhelds and mobile technologies in libraries: how the academic library is using handheld mobile technologies. 2007.

http://web.simmons.edu/ fox/pda/

Gimson, Roger (ed.). Device independence principles. 2003. W3C Working group note, 2003, 1 de septiembre.

http://www.w3.org/TR/2003/NOTE-di-princ-20030901/

Jaokar, Ajit; Fish, Tony. Mobile Web 2.0: the innovator's guide to developing and marketing next generation wireless/mobile applications. London: Futuretext, 2006. ISBN 0-9544327-6-2.

Kroski, Ellyssa. "On the move with the mobile Web: libraries and mobile technologies". Library technology reports, 2008, julio, v. 44, n. 5.

La sociedad de la información en España 2008. Madrid: Ariel, 2008. Colección Fundación Telefónica. ISBN 978-84-0808-534-8.

http://e-libros.fundacion.telefonica.com/sie08/aplicacion_sie/ParteA/datos. html

Moll, Cameron. Mobile Web design: a web standards approach for delivering content beyond the desktop. Cameron Moll, 2008. ISBN: 9780615185910.

Monteoliva, Eloísa; Pérez-Ortiz, Carlos; Repiso, Rafael. "Lectores de documentos electrónicos". El profesional de la información, 2008, julioagosto, v. 17 , n. 4 , pp. 396-402.

Mossberg, Walter S. "Shortcovers, iceberg put latest e-books on your cellphone". The Wall Street journal, 2009, 15 de enero. http://online.wsj.com/article/SB123198329968084069.html

Navegantes en la red: $10^{a}$ encuesta AIMC a usuarios de internet. Madrid: Asociación para la Investigación de los Medios de Comunicación, 2008. http://www.aimc.es/03internet/macro2007.pdf

Nkeze, Eman; Pearce, James; Womer, Matt (ed.). Device description landscape 1.0. W3C Working group note, 2007, 31 de octubre. http://www.w3.org/TR/dd-landscape

O'Reilly, Tim. "What is Web 2.0: design patterns and business models for the next generation of software". En: O'Reilly, 2005.

http://www.oreillynet.com/pub/a/oreilly/tim/news/2005/09/30/what-is-web20.html

Quitney, Janna; Rainie, Lee. The future of the internet III. Pew internet, 2008, 14 de diciembre.

http://www.elon.edu/docs/e-web/predictions/2008_survey.pdf

Rabin, Jo; McCathieNevile, Charles (ed.). Mobile Web best practices 1.0. Basic guidelines. W3C Recommendation, 2008, 29 de julio. http://www.w3.org/TR/2008/REC-mobile-bp-20080729/

Sierra, Tito; Ryan, Joseph; Wust, Markus. "Beyond OPAC 2.0: library catalog as versatile discovery platform". The Code4Lib journal, 2007, 17 de diciembre, n. 1.

http://journal.code4lib.org/articles/10

Tosete-Herranz, Francisco. "Web móvil”. Anuario ThinkEPI 2008. Barcelona: EPI SCP, 2008, pp. 174-176.

West, Mark A.; Hafner, Arthur W.; Faust, Bradley D. "Expanding access to library collections and services using small-screen devices". Information technology and libraries, 2007, 14 de agosto.

http://www.ala.org/ala/mgrps/divs/lita/ital/252006/2502jun/west.cfm

\section{Natalia Arroyo-Vázquez}

Departamento de Análisis y Estudios. Fundación Germán Sánchez Ruipérez. Plaza de España, 14. 37300

Peñaranda de Bracamonte, Salamanca

narroyo@fundaciongsr.es 